\title{
Role of Amorphous Region on the Deformation Behavior of Crystalline Polymers
}

\author{
Mari Fukuoka, ${ }^{1}$ Tetsuya Aya, ${ }^{2}$ Hiromu SAito,${ }^{1, \dagger}$ Shoji ICHIHARA, ${ }^{1}$ and Hironari SAnO ${ }^{3}$ \\ ${ }^{1}$ Department of Organic and Polymer Materials Chemistry, Tokyo University of Agriculture and Technology, \\ Koganei 184-8588, Japan \\ ${ }^{2}$ Mitsubishi Chemical Group, Science and Technology Research Center, Inc., Yokkaichi 510-8530, Japan \\ ${ }^{3}$ Mitsubishi Chemical Group, Science and Technology Research Center, Inc., Yokohama 227-0033, Japan
}

(Received January 13, 2006; Accepted January 25, 2006; Published May 17, 2006)

\begin{abstract}
As a means of better understanding the role of amorphous chains on the deformation behavior of crystalline polymers, we prepared high density polyethylene (HDPE) having various chain densities by extracting the petroleum resin dissolved in the interlamellar amorphous region of HDPE/petroleum resin blends. The small angle $\mathrm{X}$-ray scattering intensity increased by increasing the amount of petroleum resin extracted, suggesting that the density of the amorphous chain in the interlamellar amorphous region decreases with an increase in the extracted petroleum resin. The yield stress and Young's modulus decreased while the drawing property was improved by decreasing the amorphous chain density in the interlamellar amorphous region. TEM observation of the stretched specimen revealed that the decrease in overall stress is ascribed to the enhancement of the lamellar separation associated with a large void formation and lamellar deflection due to the low number density of tie chains, which act as transmitters of the external force in the interlamellar amorphous region. [doi:10.1295/polymj.PJ2005213]

KEY WORDS Crystalline Polymer / High Density Polyethylene / Amorphous Chain / TEM Observation / Deformation Behavior /
\end{abstract}

The tensile properties of crystalline polymers are elucidated by a lamellar structure of alternating crystalline and amorphous regions. ${ }^{1}$ Crystalline polymers are considered composite materials consisting of a crystalline phase with high strength and an amorphous phase with low strength. It is often reported that the modulus and yield stress simply increase as the crystallinity increases. ${ }^{2-4}$ However, the mechanical properties of crystalline polymers are not simple and can be different even if the crystallinity is the same. ${ }^{5}$ The differences in the mechanical properties are ascribed to differences in the amorphous structure by various chain conformations, e.g., an adjacent crystalline phase is linked by tie chains that are entangled with loop chains, cilia chains, or floating chains in the amorphous phase. By taking into account tie chains, the Takayanagi model has explained the modulus of the crystalline polymers and of revised ones in which the crystalline phase and amorphous phase are joined in series and/or parallel according to the morphological features of the crystalline polymers. ${ }^{6-9}$ The stress-strain relationship of crystalline polymers is predicted by a primitive molecular theory based on the change in the conformational free energy of tie chains, and the calculated results suggest that the overall stress increases with an increase in the fraction of tie chains. ${ }^{10-13}$ To date, the role of amorphous chains on the mechanical properties of crystalline polymers has not been confirmed experimentally.

In this paper, to better understand the role of amorphous chains on the deformation behavior of crystalline polymers from an experimental viewpoint, we prepared high density polyethylene (HDPE) having various amorphous chain densities by extracting the petroleum resin, which dissolves in the interlamellar amorphous region of HDPE/petroleum resin blends. After the extraction, the mechanical properties of the blends were investigated by tensile testing. The results are discussed in terms of the amorphous chain density estimated by small angle X-ray scattering (SAXS) and the deformed lamellar structure observed by transmission electron microscopy (TEM).

\section{EXPERIMENTAL}

The HDPE used was a commercial polymer supplied by Japan Polyethylene Corporation (Novatec HY540, $\left.M_{\mathrm{n}}=2.1 \times 10^{4}, T_{\mathrm{m}}=135^{\circ} \mathrm{C}\right)$. Hydrogenated $\mathrm{C} 5$ type petroleum resin (petroleum resin) was supplied by Idemitsu Kosan Co., Ltd. (Aimarb P140, $\left.M_{\mathrm{n}}=930, T_{\mathrm{g}}=90^{\circ} \mathrm{C}\right)$.

HDPE and petroleum resin were melt-mixed at $190^{\circ} \mathrm{C}$ and a screw rotation speed of $40 \mathrm{rpm}$ by using a Labo Plastomill twin-screw extruder having a screw diameter of $20 \mathrm{~mm}$ (Toyo Seiki Seisaku-sho, Ltd.). The blend specimen thus prepared was extruded at

${ }^{\dagger}$ To whom correspondence should be addressed (E-mail: hsaitou@cc.tuat.ac.jp). 
$190^{\circ} \mathrm{C}$ by using a single-screw extruder equipped with a T-die (Osada Co., Ltd.). The extruded specimen was drawn in the extruded direction into a film specimen with a thickness of $50 \mu \mathrm{m}$ by using a take-up unit at a rate of $9 \mathrm{~m} / \mathrm{min}$.

In order to extract the petroleum resin from the blend film, the film specimen was stirred with hexane at room temperature for $3 \mathrm{~d}$ and then was dried under vacuum $\left(10^{-4} \mathrm{mmHg}\right)$ for $1 \mathrm{~d}$ at room temperature to remove residual solvent. The extraction of the petroleum resin was confirmed by the weight ratio of the extracted specimen and that of a non-extracted specimen.

The film specimen was placed on a cover glass and observed under a BX-50 optical microscope (Olympus Co., Ltd.).

The SAXS measurements were performed by a NANO-Viewer (Rigaku Co., Ltd.). The radiation of a $\mathrm{Cu}$ anode was reflected from a graphite monochromator to obtain monochromatic $\mathrm{CuK} \alpha$ radiation with wavelength $\lambda=0.154 \mathrm{~nm}$. The scattered intensity was detected by an R-Axis DS3 imaging plate (Rigaku Co., Ltd.). Measurement was performed at $20^{\circ} \mathrm{C}$. The scattering profiles were corrected for background.

In order to measure the stress-strain curve, the film specimen was cut into a 50-mm long rectangle in the extruded direction (ED) and a width of $10 \mathrm{~mm}$ in the transverse direction. The stress-strain curve of the film specimen was measured by using a tensile testing machine (VES05D, Toyoseiki Co., Ltd.) at crosshead speed of $50 \mathrm{~mm} / \mathrm{min}$ at $20^{\circ} \mathrm{C}$. The stretching direction was parallel to the ED.

The melting behavior of the specimen was characterized with a Pyris 1 differential scanning calorimeter (DSC) manufactured by Perkin Elmer. The specimen was heated in the DSC from 50 to $190^{\circ} \mathrm{C}$ at a scanning rate of $10^{\circ} \mathrm{C} / \mathrm{min}$ under a nitrogen atmosphere and a DSC thermogram was recorded.

For TEM analysis, ultrathin sections were cut from the film specimen with a microtome and stained with ruthenium tetraoxide $\left(\mathrm{RuO}_{4}\right)$. The morphology was observed under a TEM (JEM1230, JEOL Co., Ltd.).

\section{RESULTS AND DISCUSSION}

When HDPE/petroleum resin blends (non-extracted blends) of low petroleum resin content (concentration of petroleum resin lower than $30 \mathrm{wt} \%$ ) were annealed above the melting point of the HDPE, no structure was seen under an optical microscope. The result suggests that HDPE and petroleum resin are miscible at low petroleum resin content. The miscibility of HDPE and petroleum resin was confirmed by melting point depression and a change of the spherulite structure with the blend composition, as exhibited in our previous paper. ${ }^{14}$ In contrast, a two-phase structure was observed when the blends of high petroleum resin content (concentration of petroleum resin higher than $30 \mathrm{wt} \%$ ) were annealed above the melting point of the HDPE. Hence, in this paper, the blends demonstrated were limited in low petroleum resin content below $30 \mathrm{wt} \%$ in which HDPE and petroleum resin are miscible.

Figure 1 shows the TEM micrographs for neat HDPE and 80/20 HDPE/petroleum resin after extraction of the petroleum resin (extracted 80/20 blend). The crystalline lamellae appear as bright stripes because the staining agent $\left(\mathrm{RuO}_{4}\right)$ cannot penetrate into the crystalline regions. In neat HDPE, the crystalline lamellae are straight and long (Figure 1a). A similar lamellar structure is seen in the extracted blends (Figure 1b). The longitudinal direction of the lamellae is perpendicular to the extruded direction (ED), suggesting that the preferential chain direction is perpendicular to the longitudinal direction of the lamellae and is parallel to the ED. The significant result here is that no void was observed in the extracted blends although the petroleum resin was extracted from the amorphous region of the blends. This suggests that

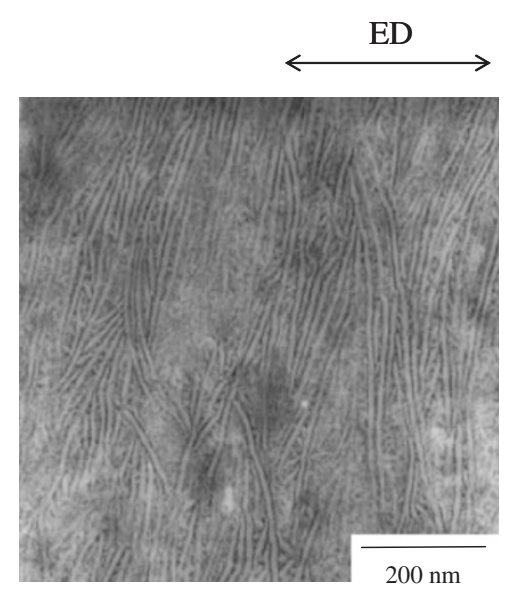

(a) neat HDPE

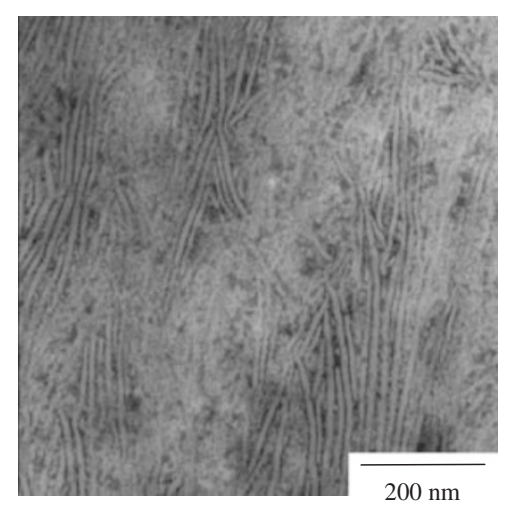

(b) extracted 80/20 HDPE / petroleum resin

Figure 1. TEM micrographs of neat HDPE and extracted 80/ $20 \mathrm{HDPE} /$ petroleum resin blend. 
the petroleum resin is miscible with HDPE and is dissolved in the interlamellar amorphous region. If the petroleum resin that is dissolved in the interlamellar amorphous region is extracted, the amorphous chain structure in the interlamellar amorphous region should be changed. The existence of the petroleum resin in the interlamellar amorphous region of the blends and the change in the amorphous chain structure by extracting the petroleum resin can be confirmed by the SAXS results, as explained below.

Figure 2 shows the SAXS profiles of non-extracted and extracted blends. Here the scattering intensity was normalized by the thickness of the film specimen. The peak position shifts only slightly with the blend composition, suggesting that the most probable nextneighbor distance of the lamellae changes only slightly with the blend composition. In contrast, the scattering intensity changes with the blend composition. The intensity decreases with an increase in the petroleum resin in the non-extracted blends (Figure 2a), while it increases in the extracted blends (Figure 2b).

In order to discuss the scattering intensity, it is convenient to employ the integrated scattering intensity,

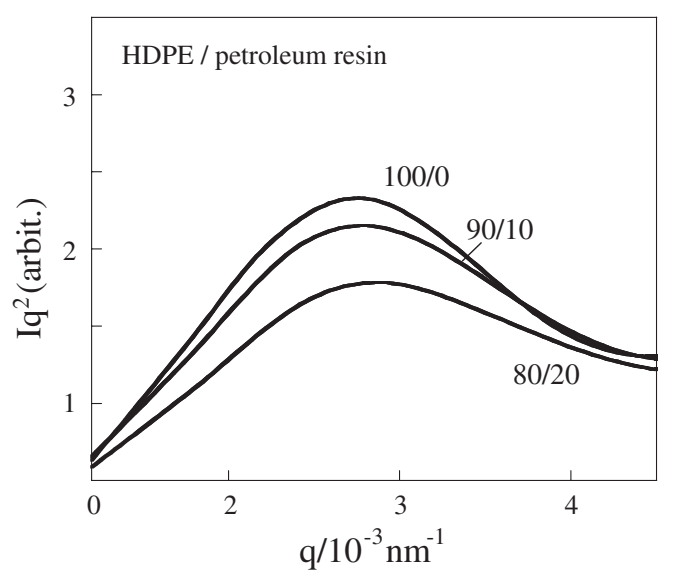

(a) non-extracted blends

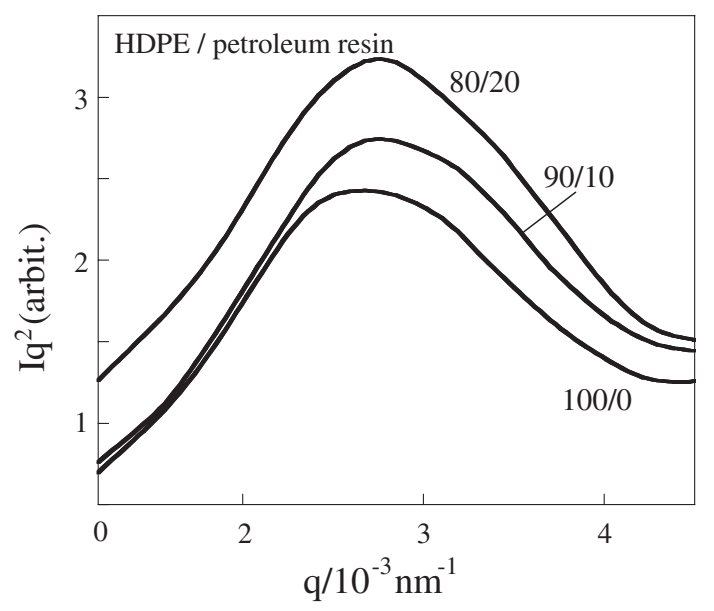

(b) extracted blends

Figure 2. SAXS profiles of HDPE/petroleum resin blends. i.e., the invariant $Q$ defined by ${ }^{15}$

$$
Q=\int_{0}^{\infty} I(q) q^{2} d q
$$

where $I$ is the X-ray scattering intensity at the scattering vector $q, q=(4 \pi / \lambda) \sin (\theta / 2), \lambda$ and $\theta$ being the wavelength of the $\mathrm{X}$-ray and the scattering angle, respectively. $Q$ becomes larger as the scattered intensity $I$ increases. Thus, the results in Figure 2 suggest that $Q$ decreases with an increase in the petroleum resin in the non-extracted blends, while it increases in the extracted blends.

Assuming that the amorphous region outside the lamellar stacks does not contribute to $Q$ at a small angle, $Q$ for an ideal two-phase system is given by ${ }^{16-18}$

$$
Q=\alpha \phi_{\mathrm{c}}\left(1-\phi_{\mathrm{c}}\right)\left(\eta_{\mathrm{c}}-\eta_{\mathrm{a}}\right)^{2}
$$

where $\alpha$ is the volume fraction of lamellar stacks within the spherulites, $\phi_{\mathrm{c}}$ is the degree of crystallinity within the lamellar stack, and $\left(\eta_{\mathrm{c}}-\eta_{\mathrm{a}}\right)$ is the difference in the electron density between the crystal and amorphous regions. The structural parameters for equation (2) are shown in Table I. The lamellar thickness $d$ and next-neighbor distance of the lamellae $L$ estimated by TEM observation changed little with blend composition, so that a ratio of these values $\phi_{\mathrm{c}}=$ $d / L$ changes little with blend composition. The overall crystallinity $\phi_{\mathrm{o}}$ obtained by DSC also changes little with blend composition, so that the $\alpha$ obtained by a ratio of $\phi_{\mathrm{c}}$ and $\phi_{\mathrm{o}}\left(\alpha=\phi_{\mathrm{c}} / \phi_{\mathrm{o}}\right)$ changes little with blend composition. Thus, the decrease of the scattering intensity and the invariant with an increase in the petroleum resin in the non-extracted blends shown in Figure $2 \mathrm{a}$ is ascribed to the decrease of $\left(\eta_{\mathrm{c}}-\eta_{\mathrm{a}}\right)$ by an increase in $\eta_{\mathrm{a}}$ due to the existence of the dissolved petroleum resin in the interlamellar amorphous region. In contrast, the increase of the scattering intensity with the petroleum resin in the extracted blends, shown in Figure $2 b$, is ascribed to the increase of $\left(\eta_{\mathrm{c}}-\eta_{\mathrm{a}}\right)$ by a decrease in $\eta_{\mathrm{a}}$ with the petroleum resin due to the decrease of the chain density in the interlamellar amorphous region by extracting the petroleum resin. This suggests that the number density of the molecular chain in the interlamellar amorphous region decreases by extracting the petroleum resin,

Table I. Structural parameters of HDPE/petroleum resin blends

\begin{tabular}{cccc}
\hline & $100 / 0$ & $90 / 10$ & $80 / 20$ \\
\hline$Q$ (arbit.) & 5.6 & 6.0 & 6.6 \\
$\phi_{\mathrm{o}}(\%)$ & 64.2 & 61.6 & 61.7 \\
$\phi_{\mathrm{c}}(\%)$ & 57.7 & 53.8 & 53.5 \\
$\alpha$ & 0.90 & 0.87 & 0.87 \\
$\left(\eta_{\mathrm{c}}-\eta_{\mathrm{a}}\right)^{2}$ & 2.6 & 2.7 & 3.0 \\
\hline
\end{tabular}




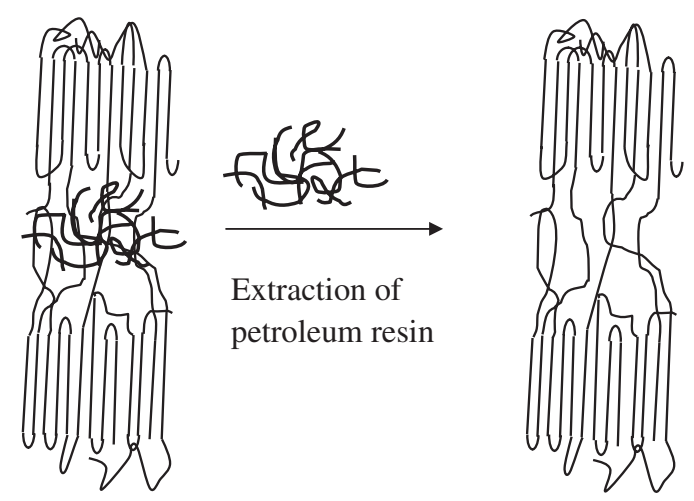

Figure 3. Schematic illustrations of HDPE/petroleum resin blends before and after extraction of the petroleum resin.

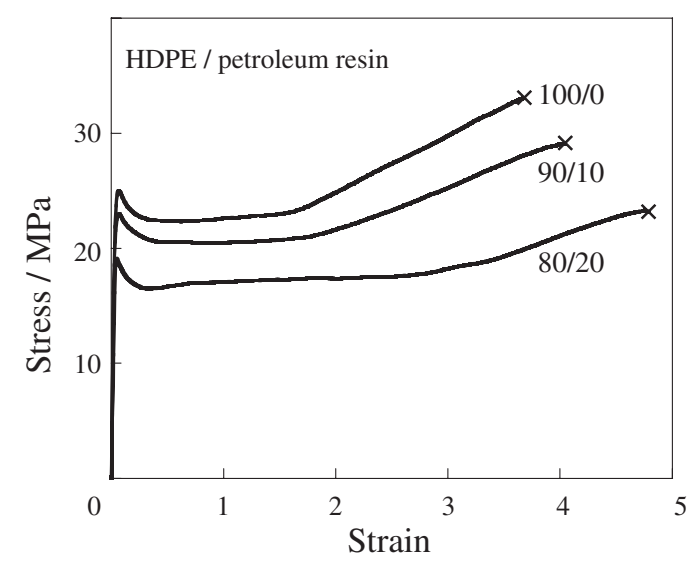

Figure 4. Stress-strain curves of extracted HDPE/petroleum resin blends.

as schematically shown in Figure 3. The decrease of the amorphous chain density is larger with an increase in the extracted petroleum resin. The decrease of the amorphous chain density may correspond to the decrease of the number density of tie chains and entanglements in the interlamellar amorphous region.

Figure 4 shows the stress-strain curves of neat HDPE and extracted blends. Here, the stretching direction is parallel to the extruded direction (ED) and is perpendicular to the longitudinal direction of the lamellae. Initially, the stress rises in a linear manner with increased strain; then the stress reaches a maximum at the yield point. After the subsequent fall and plateau in the stress by strain softening, the stress again increases by strain hardening up to the breaking point. The shape of the stress-strain curves of the extracted blends are similar to that of neat HDPE, but the overall stress such as yield stress and stress at break decreases with an increase in the fraction of the extracted petroleum resin. When the stretching direction is perpendicular to the longitudinal direction of the lamellae, the neighboring lamellae are separated in association with the fragmentation of the stacked

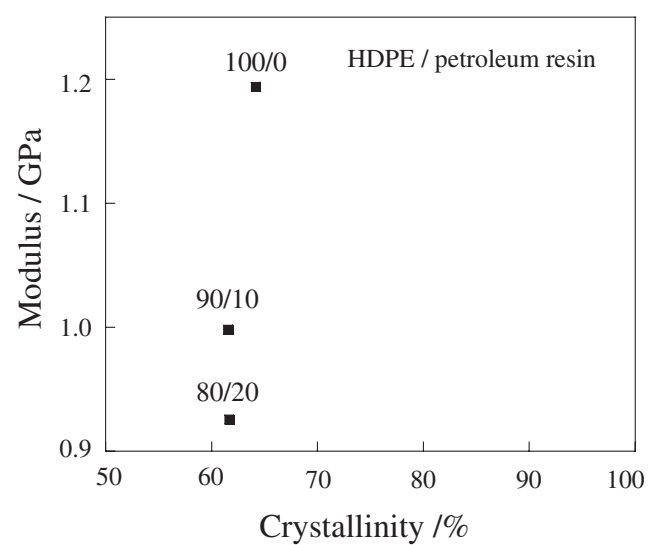

Figure 5. Modulus of extracted HDPE/petroleum resin blends as a function of degree of crystallinity.

lamellae by chain slip in the amorphous region. ${ }^{19}$ The lamellar separation causes tie chains to contribute a positive stress and to resist further lamellar separation. ${ }^{20}$ Thus, the decrease of overall stress by extracting the petroleum resin is ascribed to the decrease of the number density of tie chains in the interlamellar amorphous region.

The modulus of the film specimen was obtained by the initial slopes of the stress-strain curves in Figure 4. The modulus of neat HDPE and the extracted blends are shown in Figure 5 as a function of crystallinity. Here, the crystallinity is overall crystallinity estimated by DSC. In general, no difference of the modulus is expected when the crystallinity and the lamellar shape are the same in the same material. However, the modulus decreases with an increase in the fraction of the extracted petroleum resin although the crystallinity is almost the same. The change of the modulus is also ascribed to the decrease of the chain density in the interlamellar amorphous region by the extraction of the petroleum resin; i.e., the force transmitted in the interlamellar amorphous region decreases due to the decrease of the number density of tie chains and entanglements. ${ }^{6-9}$

Figure 6 shows TEM micrographs for neat HDPE and extracted $80 / 20$ blend stretched at a draw ratio of $\lambda=2$ in which the strain softening proceeds after yielding and before the start of strain hardening. Distorted voids having a size of $10-30 \mathrm{~nm}$ are seen as bright ellipsoids in the interlamellar amorphous region of the neat HDPE (Figure 6a). The lamellae are tilted and splayed apart in the area around the voids. When the lamellae are stretched perpendicular to the longitudinal direction, lamellar separation occurs by external force. Because of the lamellar separation, the interlamellar amorphous region is opened and then the voids are developed. The lamellae are tilted and splayed apart due to the development of the voids and the stress concentration by the existence of the 


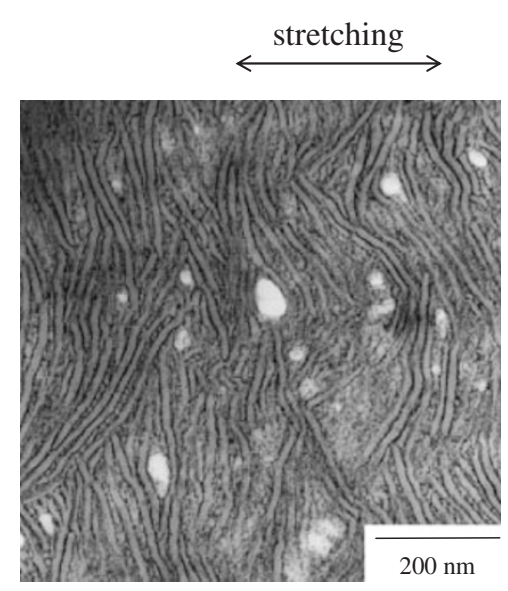

(a) neat HDPE

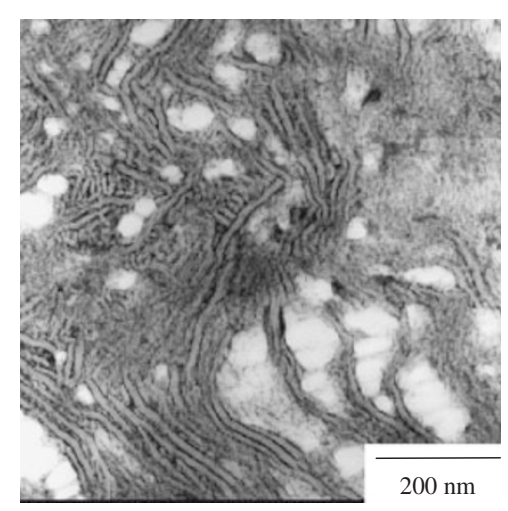

(b) extracted 80/20 $\mathrm{HDPE} /$ petroleum resin

Figure 6. TEM micrographs of HDPE lamellae stretched at a stretching rate of $\lambda=2$.

voids. The voids in the extracted blends are larger and heterogeneously distributed compared with those in the neat HDPE (Figure 6b). The lamellae in the area around the voids are deflected. Since the void is developed by the opening and splaying of the interlamellar amorphous region, the large void in the extracted blends is induced by the enhancement of the lamellar separation due to the decrease of the number density of tie chains and entanglements, which act as transmitters of the external force. The heterogeneously distributed voids may be ascribed to the heterogeneous distribution of the chain density in the interlamellar amorphous region. Owing to the heterogeneous distribution of the chain density and large void formation, a large stress concentration is induced. Because of the large stress concentration, the lamellae are deflected. Thus, the interlamellar amorphous region is opened and the lamellae are separated during the stretching in the strain softening process associated with void formation and lamellar deflection caused by the low number density of the amorphous chains, as schematically shown in Figure 7. This may cause the decrease of the overall stress shown in Figure 4.

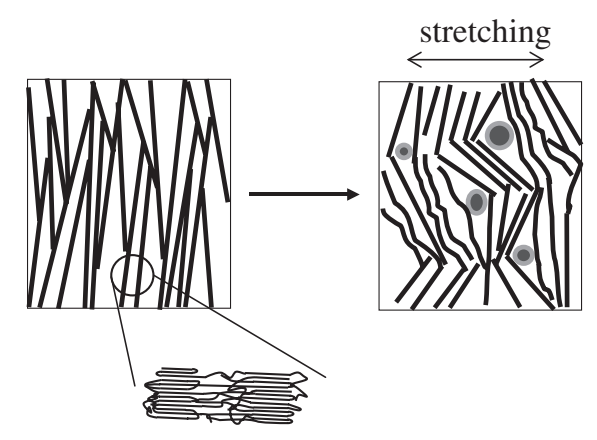

(a) neat HDPE

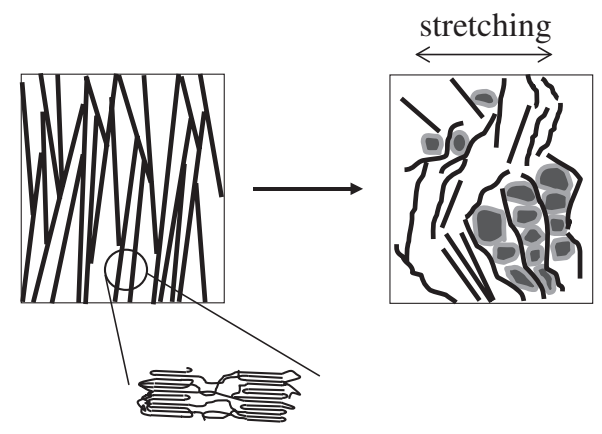

(b) extracted blend

Figure 7. Schematic illustrations of the deformation mechanism of neat HDPE and extracted HDPE/petroleum resin blends.

\section{CONCLUSION}

By extracting the petroleum resin from the miscible blends of HDPE and petroleum resin, HDPE film having a variety of chain densities in the interlamellar amorphous region was obtained. As the number density of tie chains and entanglements decreases with a decrease in the chain density, the overall stress decreases. This may be ascribed to the enhancement of the lamellar separation associated with large void formation and lamellar deflection caused by the low number density of tie chains, which act as transmitters of the external force.

\section{REFERENCES}

1. "Materials Science and Technology: Structure and Properties of Polymers," R. W. Cahn, P. Haasen, and E. J. Kramer, Ed., VCH Publishers Inc., Cambridge, 1993.

2. P. D. Davides, H. I. Waterman, and I. B. Westerdijk, J. Polym. Sci., 59, 389 (1962).

3. S. Fisher and N. Brown, J. Appl. Phys., 44, 4322 (1973).

4. R. Popli and L. Mandelkern, J. Polym. Sci., Part B: Polym. Phys., 25, 441 (1987).

5. S. Ichihara, Kobunshi Ronbunshu, 55, 7 (1998).

6. M. Takayanagi, I. Imada, and T. Kajiyama, J. Polym. Sci., Part C, 15, 263 (1966).

7. A. G. Gibson, G. R. Davies, and I. M. Ward, Polymer, 19, 683 (1978). 
8. K. Nitta and M. Takayanagi, J. Polym. Sci., Part B: Polym. Phys., 38, 1037 (2000).

9. I. M. Ward and J. Sweeney, "An Introduction to the Mechanical Properties of Solid Polymers," 2nd ed., John Wiley \& Sons, Ltd., West Sussex, England, 2004.

10. D. J. Lohse and R. J. Gaylord, Polym. Eng. Sci., 18, 512 (1978).

11. R. J. Gaylord, Polym. Eng. Sci., 19, 955 (1979).

12. M. Takayanagi and K. Nitta, Macromol. Theory Simul., 6, 181 (1997).

13. K. Nitta, Comput. Theor. Polym. Sci., 9, 19 (1999).
14. T. Aya, M. Fukuoka, H. Saito, and S. Ichihara, Kobunshi Ronbunshu, 62, 579 (2005).

15. O. Glatter and O. Kratky, "Small Angle X-ray Scattering," Academic Press, London, 1982.

16. G. R. Strobl, M. J. Schneider, and I. G. Voigt-Martin, J. Polym. Sci., Part B: Polym. Phys., 18, 1361 (1980).

17. H. Saito and B. Stühn, Macromolecules, 27, 216 (1994).

18. C. H. Lee, H. Saito, T. Inoue, and S. Nojima, Macromolecules, 29, 7034 (1996).

19. H. Zhou and G. L. Wilkes, J. Mater. Sci., 33, 287 (1998).

20. K. Nitta, Macromol. Symp., 170, 311 (2001). 